\title{
Desigualdades Regionais de Renda e as Variações nos Determinantes do Crescimento Econômico entre os Estados Brasileiros - no Período de 1995 a 2004
}

Melquisedeque Tavares Oliveira ${ }^{1}$

Adriano Nascimento da Paixão ${ }^{2}$

Resumo: O objetivo deste trabalho foi descrever e analisar as diferenças regionais nos determinantes da renda, indicados pelo modelo de Mankiw, Romer e Weil (1992), e contrapô-las com as disparidades de renda per capita que existem entre as regiões brasileiras. A investigação econométrica realizada neste estudo está baseada em modelos de regressão com dados em painel, referentes ao período 1995-2004. Foram consideradas todas as unidades federativas brasileiras, e os dados secundários foram obtidos no Instituto de Pesquisa Econômica Aplicada - IPEA. Uma das conclusões obtidas é que existem no Brasil consideráveis variações regionais tanto nos efeitos quanto nas dotações das variáveis consideradas determinantes da renda per capita de longo prazo. Esses dois tipos de variações reforçam, de forma conjunta, as desvantagens das regiões mais pobres (Norte e Nordeste), o que leva a concluir que existem grandes diferenças nas condições estruturais das economias dos estados e macrorregiões do país.

Palavras-Chave: Modelos de Crescimento Econômico; Determinantes do Crescimento Econômico; Desigualdades Regionais.

JEL: C13, C23, O11, O41, R11

1 Economista e Mestre em Desenvolvimento Regional pela UFT.

2 Economista e Mestre em Economia Aplicada pela UFPB, Doutor em Economia Aplicada pela UFV. 
Abstract: The aim of this study was to describe and analyze regional differences in the determinants of income, indicated by Mankiw, Romer and Weil (1992), and confrota them with disparities in per capita income that exist between regions. The econometric investigation in this study is based on regression models with panel data for the period 1995-2004. All Brazilian states were considered, and the secondary data were obtained from the Institute of Applied Economic Research - IPEA. One of the conclusions is that in Brazil there are considerable regional variations in the effects and in the appropriations of the variables considered determinants of per capita income of the long run. These two variations reinforce, jointly, the disadvantages of the poorest regions (North and Northeast), which leads to the conclusion that there are large differences in the structural conditions of the economies of states and geographical regions of the country.

Keywords: Models of Economic Growth; Determinants of Economic Growth; Regional Inequalities.

JEL: C13, C23, O11, O41, R11

\section{Introdução}

No Brasil, foram aplicados testes para a hipótese de convergência de renda para os municípios e estados brasileiros, no entanto, muitos desses trabalhos concluíram que ocorreu convergência apenas de forma condicionada a determinadas variáveis, por exemplo: tecnologia, investimentos em capital físico e em capital humano. Dentre os estudos com conclusões neste sentido apontam-se os seguintes: Ferreira (1996), Ferreira (2000), Azzoni et al (2000), Chaves (2003), Chagas e Toneto Jr. (2003), Azzoni et al. (2004), Silveira-Neto e Azzoni (2005) e Resende e Figueiredo (2005) e Ribeiro et al (2013).

A partir de Mankiw et al (1992), pode se considerar as referidas variáveis condicionantes como sendo determinantes da renda per capita de longo prazo, portanto, é possível entender esses determinantes como condições estruturais de cada economia, isto é, que envolvem elementos que permanecem por longos períodos, e, portanto, diferenças em seus níveis implicam em gaps de renda permanentes.

À vista disso, as disparidades regionais de renda persistentes podem ser reflexos de diferenças nos citados determinantes de longo prazo, entre as regiões, ou seja, de suas condições estruturais, por não mudarem facilmente em períodos curtos ou médios.

Desta maneira, fica evidente a pertinência de análises que centralizem esses 
determinantes, descrevendo suas variações regionais e confrontado-as com as características da distribuição da renda per capita das regiões brasileiras, com intuito de compreender as razões das desigualdades regionais e para visualizar perspectivas futuras em relação à evolução nesse quadro.

Adicionalmente, observam-se, na literatura empírica brasileira, diversos indícios indiretos de diversidade nas características das regiões com respeito aos seus determinantes da renda de longo prazo, porém, ainda é relevante que se realize um estudo que descreva diretamente as diferenças dessas características entre as regiões mais pobres e as regiões mais ricas, e como e se essas diferenças ajudam a explicar as disparidades de renda entre tais regiões. No presente estudo, esse será o objeto sob análise, sendo que as unidades regionais observadas em primeiro plano serão as unidades federativas brasileiras.

Portanto, o objetivo geral desse estudo é descrever e analisar as diferenças regionais nos determinantes da renda indicados pelo modelo de Mankiw, Romer e Weil (1992), e contrapô-las com as disparidades de renda per capita que existem entre as regiões brasileiras.

Além desta introdução, este trabalho possui mais quatro seções. Na seção 01, apresenta-se o referencial teórico, que serve como base para todas as análises e procedimentos econométricos deste trabalho. Na sequência, a seção 02 apresenta os métodos e os materiais utilizados neste estudo, enquanto que a seção 03 aplica tais métodos e materiais, de onde se obtém os resultados e discussões pertinentes. Já as conclusões obtidas através das análises dos resultados estão na última seção.

\section{Referencial Teórico}

\subsection{Uma aproximação empírica para o Modelo de Solow ampliado com capital humano ${ }^{1}$}

O Modelo de Solow ampliado com capital humano (MRW) proposto por Mankiw, Romer e Weil (1992) incluiu o capital humano no modelo de Solow tradicional (1956), e seus autores concluíram que essa inclusão melhorava o desempenho empírico do modelo. Segundo Jones (2000), a referida inclusão equivale a reconhecer as diferenças nos níveis de instrução e qualificação que existem entre diferentes economias.

Desse modo, o capital humano foi incorporado na função de produção representado por H, que também é conhecido como trabalho qualificado. Sendo 
assim, a função de produção assume a seguinte forma:

$$
Y_{t}=K_{t}^{\alpha} H_{t}^{\beta}\left(A_{t} L_{t}\right)^{1-\alpha-\beta}
$$

Em que, $\mathrm{K}$ representa o estoque total de capital físico, $\mathrm{Y}$ o produto total, $\mathrm{L}$ o número de trabalhadores na economia (trabalho) e $\mathrm{A}$ a tecnologia aumentadora de trabalho.

Dividindo (1) por $\left(A_{t} L_{t}\right)$ obtém-se a função de produção por trabalhador em unidades efetivas de trabalho:

$$
\hat{\boldsymbol{y}}=\hat{\boldsymbol{k}}^{\alpha} \hat{\boldsymbol{h}}^{\beta}
$$

A acumulação de $\mathrm{k}$ e $\mathrm{h}$ ocorrem de acordo com as seguintes equações

$$
\begin{aligned}
& \mathrm{k}_{\mathrm{t}}=\mathrm{s}_{\mathrm{k}} \mathrm{y}_{\mathrm{t}}-(\mathrm{n}+\mathrm{g}+\mathrm{d}) \mathrm{k}_{\mathrm{t}} \\
& h_{t}=s_{h} y_{t}-(n+g+d) h_{t}
\end{aligned}
$$

Em que $s_{h}$ representa a fração da renda que é poupada para o investimento em capital humano; $s_{k}$ é a fração da renda que é poupada para investimento em capital físico; $d$ representa a depreciação que incide sobre o estoque total de capital físico; $\dot{k}$ significa a variação no estoque de capital físico; enquanto $\dot{h}$ significa a variação no estoque de capital humano; o crescimento populacional é representado por $n$; e o progresso tecnológico é expresso por $\mathrm{g}$.

O estado estacionário será obtido quando $\dot{\mathrm{k}}=0$ e $\dot{h}=\mathrm{o}$ nas equações (3) e (4), o que resulta em um sistema de equações da onde é possível isolar he k , obtendo assim seus valores no estado estacionário:

$$
\begin{aligned}
k^{*} & =\left(\frac{s_{k}^{1-\beta} s_{h}^{\beta}}{n+g+\delta}\right)^{\frac{1}{(1-\alpha-\beta)}} \\
h^{*} & =\left(\frac{s_{k}^{\alpha} s_{h}^{1-\alpha}}{n+g+\delta}\right)^{\frac{1}{(1-\alpha-\beta)}}
\end{aligned}
$$


Sendo que essas expressões podem ser substituídas na função de produção em unidades efetivas de trabalho (2), definindo o produto por unidades efetivas de trabalho $\hat{y}$, e, por conseqüência, definindo produto por trabalhador e produto per capita:

$$
y^{*}=\left(\frac{s_{k}}{n+g+d}\right)^{\frac{\alpha}{1-\alpha-\beta}}\left(\frac{s_{h}}{n+g+d}\right)^{\frac{\beta}{1-\alpha-\beta}} A_{0} e^{g t}
$$

Em síntese, diferenças persistentes no produto per capita entre regiões são oriundas de diferenças em: $A_{o}, s_{h}, s_{k}, \mathrm{n}, \mathrm{g}$, d. Isto, depois da trajetória ao nível de longo prazo.

Na sequência, aplicam-se logaritmos naturais aos dois lados da equação 07, para obter:

$$
\ln \left(y^{*}\right)=\ln A_{0}+g t-\left(\frac{\alpha+\beta}{1-\alpha-\beta}\right) \ln (n+g+d)+\left(\frac{\alpha}{1-\alpha-\beta}\right) \ln (s)+\left(\frac{\beta}{1-\alpha-\beta}\right) \ln \left(s_{h}\right)
$$

Mankiw et al (1992) sugerem que:

$$
\ln A=a+\varepsilon
$$

O que resulta na equação (9) que representa a variação da renda per capita, no estado estacionário, entre diferentes economias e será estimada por este trabalho.

$$
\ln \left(y^{*}\right)=a+g t+\left(\frac{\alpha}{1-\alpha-\beta}\right) \ln (s)+\left(\frac{\beta}{1-\alpha-\beta}\right) \ln \left(s_{h}\right)-\left(\frac{\alpha+\beta}{1-\alpha-\beta}\right) \ln (n+g+d)+\varepsilon
$$

Sob a notação econométrica, e considerando as proxies apresentadas na metodologia, as regressões estimadas aqui correspondem à equação (10).

$\ln \left(\right.$ PIBpc $\left._{i t}\right)=\beta_{0}+\beta_{1} \ln \left(\right.$ energind $\left._{i t}\right)+\beta_{2} \ln \left(\right.$ escolaridade $\left._{i t}\right)-\beta_{3} \ln \left(\right.$ crescpop $\left._{i t}\right)+\varepsilon_{i t}$ 
Além disso, uma estimação conforme a seguinte especificação também foi testada:

$\ln \left(P I B p c_{i t}\right)=\beta_{0}+\beta_{1} \ln \left(\right.$ energcom $\left._{i t}\right)+\beta_{2} \ln \left(\right.$ escolaridade $\left._{i t}\right)-\beta_{3} \ln \left(\right.$ crescpop $\left._{i t}\right)+\varepsilon_{i t}$

Cabe ressaltar que aplicação de logaritmos naturais em todas as variáveis do modelo resulta que o mesmo se torna linear tanto nos parâmetros quanto nas variáveis, além de que esse tipo de modelo também conhecido como "log-log" estima coeficientes que são as elasticidades da variável dependente em relação a cada variável explicativa.

\section{Metodologia}

\subsection{Variáveis utilizadas e base de dados $^{2}$}

A investigação econométrica realizada neste estudo está baseada em modelos de regressão com dados em painel. Foram consideradas todas as unidades federadas brasileiras no período 1995-2004, e os dados secundários foram obtidos no Instituto de Pesquisa Econômica Aplicada - IPEA.

O ano final do período 1995-2004 foi escolhido devido ao fato de que as séries para as variáveis "consumo de energia elétrica industrial" e "consumo de energia elétrica comercial" possuírem dados somente até o ano de 2004. Já o ano inicial do referido período foi escolhido por que a variável "média de anos de estudo" referente ao estado do Tocantins só possuía uma série contínua a partir de 1994 .

As variáveis utilizadas na parte de estatística descritiva e na parte econométrica deste estudo estão descritas no quadro 01, juntamente com os seus respectivos sinais esperados, com base no referencial teórico. 
OLIVEIRA, M. T.; PAIXÃO, A. N. da. Desigualdades Regionais de Renda e as Variações nos Determinantes do....

QUADRO 1 - DESCRIÇÃO E SINAIS ESPERADOS DAS VARIÁVEIS

\begin{tabular}{|c|c|c|c|}
\hline Variável & Proxy Utilizada & $\begin{array}{c}\text { Sinal } \\
\text { esperado }\end{array}$ & Fonte \\
\hline $\begin{array}{c}\text { Produto } \\
\text { per capita } \\
\text { (variável } \\
\text { dependente) }\end{array}$ & PIB per capita de cada UF & $\begin{array}{c}\text { Não se } \\
\text { aplica }\end{array}$ & IPEA \\
\hline $\begin{array}{c}\text { Capital } \\
\text { Humano }\end{array}$ & $\begin{array}{c}\text { Média de anos de estudo } \\
\text { (escolaridade) da população } \\
\text { com 25 anos ou mais de idade. }\end{array}$ & + & IPEA \\
\hline $\begin{array}{c}\text { Consumo de energia elétrica } \\
\text { per capita } \\
\text { industrial (medido em mega- } \\
\text { watts-hora) dividido pela } \\
\text { população de cada UF. }\end{array}$ & $\begin{array}{c}\text { Consumo de energia elétrica } \\
\text { comercial (medido em mega- } \\
\text { watts-hora) dividido pela } \\
\text { população de cada UF. }\end{array}$ & IPEA \\
\cline { 2 - 5 } & $\begin{array}{c}\text { Taxa de crescimento da } \\
\text { população }\end{array}$ & IPEA \\
$\begin{array}{c}\text { Taxa de } \\
\text { efetiva do } \\
\text { per capital }\end{array}$ & IPEA \\
\hline
\end{tabular}

Fonte: Elaboração própria do autor com base na literatura pesquisada

\subsection{Algumas considerações sobre estimação com dados em painel}

Como foi afirmado na seção anterior, foram realizadas, neste estudo, regressões econométricas baseada no Modelo MRW, a partir de dados em painel. Estimações com esse tipo de dados tratam de variáveis que variam entre as unidades observadas $\mathbf{i}$, e entre diferentes períodos $t$.

Dessa forma, busca-se estimar, basicamente, uma regressão com a seguinte descrição:

$$
y_{i t}=x_{i t} \beta+\varepsilon_{i t}
$$

Em que $\mathrm{i}=1,2,3, \ldots, \mathrm{n} ; \mathrm{t}=1,2,3, \ldots \mathrm{T} ; y_{i t}$ é a variável dependente; $x_{i t}$ é um vetor 
de variáveis explicativas de tamanho $1 x K$; $\beta$ é um vetor de parâmetros de tamanho $K x_{1} ; \varepsilon_{i t}$ são os erros aleatórios. Podendo existir nesta regressão um termo adicional denominado efeito não-observado $c_{i}$ que varia entre as unidades observacionais.

Para o caso de uma regressão que apresente heterocedasticidade e autocorrelação simultaneamente, uma alternativa é utilizar a regressão de Prais-Winsten para corrigir tais contradições às premissas do modelo clássico de regressão linear, pois segundo Greene (2003), a transformação Prais-Winsten remove a autocorrelação e a heterocedasticidade presentes nos dados utilizados.

Sendo que, para alcançar o referido resultado, a transformação Prais-Winsten transforma as variáveis $\mathrm{Y}$ e X multiplicando as observações de cada variável presente no modelo da seguinte forma: $Y_{1} \sqrt{1-\rho^{2}}$ e $X_{1} \sqrt{1-\rho^{2}}$.

O método de Efeitos fixos é uma das alternativas viáveis quando existe $\boldsymbol{c}_{\boldsymbol{i}}$ , e a correlação entre $c_{i}$ e qualquer variável explicativa é diferente de zero. Pois, este método consiste em tentar eliminar o efeito não-observado $c_{i}$, por meio do seguinte procedimento: primeiro, calcula-se a média dos valores observados em diferentes pontos do tempo para a variável dependente, as variáveis explicativas e o termo de erro, o que resulta em:

$$
\overline{\mathrm{y}}_{i}=\overline{\mathrm{x}}_{i} \beta+c_{i}+\bar{\varepsilon}_{i}
$$

Em seguida, é subtraído (3) de (2) para cada , da seguinte forma:

$$
\mathrm{y}_{i t}-\overline{\mathrm{y}}_{i}=\left(\mathrm{x}_{i t}-\overline{\mathrm{x}}_{i}\right) \beta+\left(c_{i}-c_{i}\right)+\left(\varepsilon_{i t}-\bar{\varepsilon}_{i}\right)
$$

Assim, $\mathbf{c}_{\mathrm{i}}$ é eliminado, e a equação pode assumir a seguinte forma:

$$
\ddot{\mathrm{y}}_{i t}=\ddot{\mathrm{x}}_{i t} \beta+\ddot{\varepsilon}_{i t}
$$

No entanto, este estimador só será consistente caso a hipótese de exogeneidade estrita não seja violada.

Já o modelo de Efeitos aleatórios é utilizado também no caso de existir $\mathbf{c}_{\mathrm{i}}$. Este método compõe um novo termo a partir da seguinte estrutura: $\mathrm{v}_{i t}=c_{i}+\varepsilon_{i t}$.

No entanto, isto só é possível caso três suposições sejam atendidas: a hipótese de exogeneidade estrita não seja violada; não exista correlação entre o efeito 
não-observado $\boldsymbol{c}_{i}$ e as variáveis explicativas (principal diferença em relação ao modelo de efeitos fixos); e $c_{i}$ seja homocedástico.

Assim,

$$
\mathrm{y}_{i t}=\mathrm{x}_{i t} \beta+\mathrm{v}_{\text {it }}
$$

Com $\mathrm{v}_{i t}=c_{i}+\varepsilon_{i t}$.

Diante de todos os métodos apresentados, são necessários critérios objetivos para que se viabilize a escolha do método mais adequado para cada conjunto de dados analisado. Assim, o teste de Hausman (1978) permite saber se a possibilidade de correlação entre $\mathrm{c}_{\mathrm{i}}$ e $\mathrm{x}_{i}$ é verdadeira ou falsa em cada conjunto de dados. Caso a possibilidade seja verdadeira o método de efeitos fixos é o mais indicado, caso seja falsa o método de efeitos aleatórios é o mais adequado.

Já o teste de LM de Breusch e Pagan ${ }^{3}$ testa a hipótese de a variância de $\mathbf{c}_{\mathrm{i}}$ ser nula, o que equivale à inexistência de heterogeneidade não observada no modelo estimado. Caso esta hipótese seja confirmada pelo resultado do referido teste, as estimativas de MQO se mostram mais eficientes que as demais.

\subsection{Considerações sobre a utilização dos gráficos $\underset{-P \operatorname{Box}}{\text { B }}$}

Frente ao objetivo de analisar o padrão das diferenças de dotação de variáveis entre os diferentes conjuntos de estados, de cada macrorregião brasileira, é interessante utilizar um instrumento gráfico denominado Box-Plot para gerar relevantes informações em relação ao cenário regional da economia brasileira.

A ferramenta Box-Plot possibilita sintetizar características importantes de um conjunto de dados, por exemplo: assimetria dos dados, primeiro quartil, mediana (que é o segundo quartil) e o terceiro quartil.

Além de tudo, é possível, com esse instrumento, complementar as análises acerca da dispersão dos dados de cada região por meio da visualização da presença ou ausência de valores atípicos, já que estes têm o potencial de afetar uma medida numérica de dispersão. Inclusive, esta maneira de apresentação possibilita perceber qual é a dispersão dos dados adjacentes na ausência de um eventual valor atípico. 


\subsection{Estatísticas descritivas}

\subsubsection{Disparidades estaduais de renda per capita: compa- rativo entre o ano inicial e o ano final do período} FIGURA 1 - BOX-PLOT - PIB PER CAPITA DAS MACRORREGIÕES - ANO 1995
(ACIMA) E 2004 (ABAIXO)
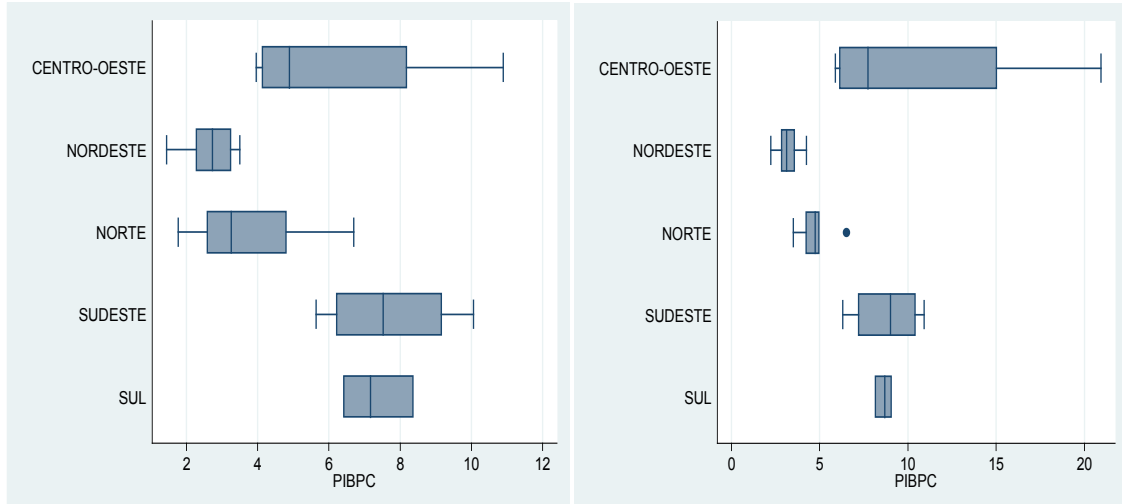

Fonte: Elaboração própria com base nos dados do IPEA, 2013.

A partir da figura 01, é possível observar, para o PIB per capita de 1995, três níveis claramente divididos, sendo que, Sudeste e Sul estão no nível mais alto, Centro-Oeste em um nível intermediário, e Norte e Nordeste no nível mais baixo. Pois, as medianas do Sudeste e do Sul são claramente superiores às medianas das outras regiões. Ademais, observa-se que as medianas dessas duas regiões são próximas entre si, com pequena vantagem do Sudeste.

Por outro lado, em 2004, visualizam-se, para essa variável, dois níveis claramente divididos, sendo que, Sudeste, Sul e Centro-Oeste estão no nível mais alto, enquanto Norte e Nordeste estão no nível mais baixo. Isso ocorreu por que houve, ao longo desse período, uma aproximação do Centro-Oeste em relação às duas primeiras macrorregiões, o que pode ser ilustrado pela maior proximidade da mediana dessa macrorregião com a medida correspondente das mais bem colocadas, na comparação com o ano inicial da série.

Já o Norte e o Nordeste continuaram nas últimas posições, sendo que suas desvantagens em relação às outras macrorregiões, em 2004, estão maiores do que em 1995. 


\subsubsection{Disparidades estaduais de escolaridade: comparati- vo entre o ano inicial e o ano final da série}

FIGURA 2 - BOX-PLOT - ESCOLARIDADE DAS MACRORREGIÕES - ANO 1995 (ACIMA) E 2004 (ABAIXO)
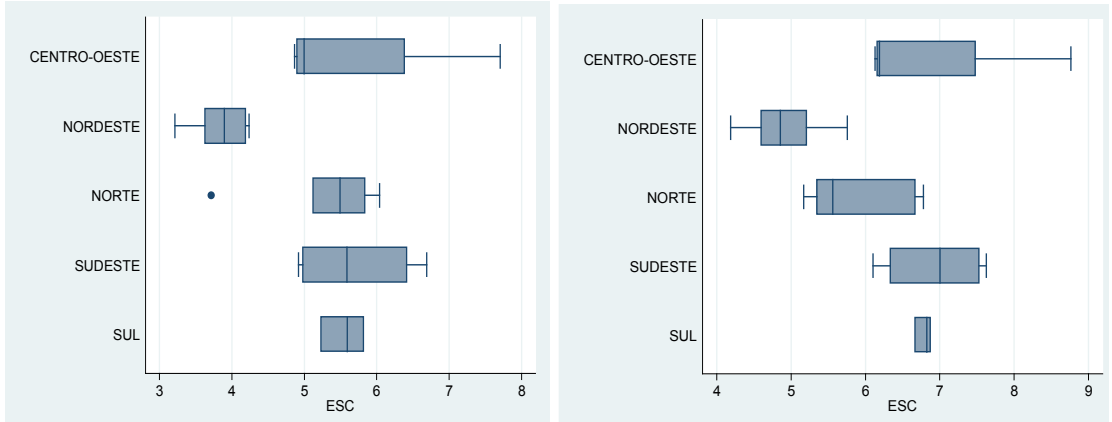

Fonte: Elaboração própria com base nos dados do IPEA, 2013.

Pela figura 02 que o Sul e o Sudeste lideravam, em 1995, com medianas muito próximas entre si. Seguidos pelo Norte, que possuía uma mediana superior ao Centro-Oeste. Enquanto que o Nordeste tinha todos os seus valores, para essa variável, inferiores a todos os valores adjacentes das outras regiões do país, apenas um valor atípico inferior do Norte é superado por algum estado nordestino.

Da mesma forma que em 1995, as regiões Sul e Sudeste continuaram, em 2004, mais favorecidas em relação à escolaridade, com medianas muito próximas entre si, enfatiza-se. Sendo que o Sul apresenta uma dispersão muito menor que o Sudeste, que, por sua vez, possui a maior mediana do Brasil para a variável proxy de escolaridade, utilizada no presente estudo.

Adicionalmente, constata-se que a mediana do Centro-Oeste, relacionada à variável escolaridade, ultrapassou a mediana do Norte ao longo do ínterim estudado. 


\subsubsection{Disparidades estaduais de consumo de energia industrial per capita: comparativo entre o ano inicial e o ano final do período}

FIGURA 3 - BOX-PLOT - CONSUMO DE ENERGIA INDUSTRIAL PER CAPITA
- ANO 1995 (ACIMA) E 2004 (ABAIXO)
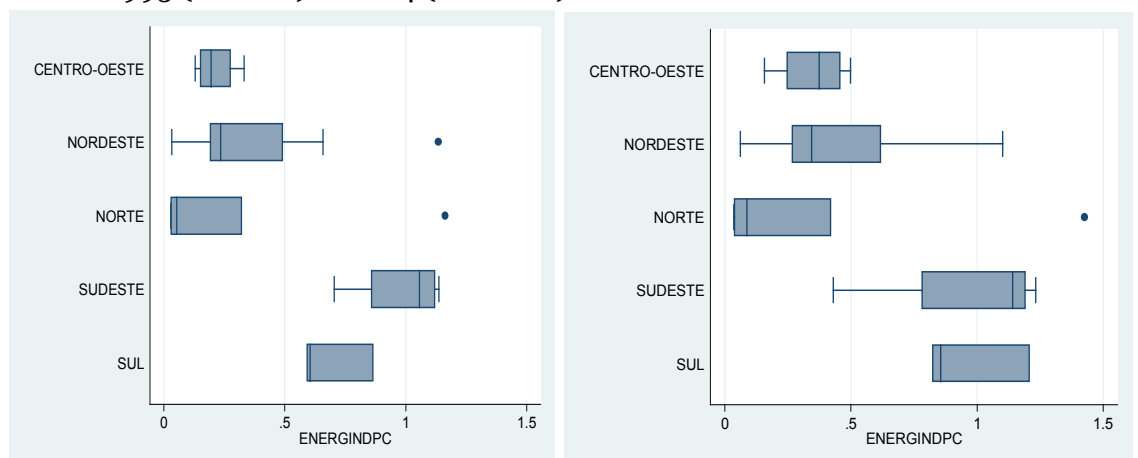

Fonte: Elaboração própria com base nos dados do IPEA, 2013.

A análise da figura 3 permite concluir que o conjunto de dados do Sudeste aparecia com grande vantagem em relação aos demais no ano de 1995, o que é ratificado pela grande diferença da sua mediana em relação às demais regiões, e pelo fato do primeiro quartil do Sudeste está muito próximo ao terceiro quartil do Sul, segunda macrorregião com maior vantagem nessa variável. Além disso, todas as observações do Sudeste são maiores que os valores adjacentes das macrorregiões Nordeste, Centro-Oeste, e Norte. Somente valores atípicos dessas macrorregiões superam os valores do Sudeste.

Ainda pela figura 3, percebe-se que houve entre 1995 e 2004 um movimento que conduziu a um maior nivelamento das macrorregiões Sudeste e Sul, o que pode ser ilustrado pela proximidade entre os primeiros quartis das duas regiões, o mesmo ocorre com os terceiros quartis. Além disso, uma diferença entre essas duas regiões é a dispersão dos dados. Pois, esta é muito maior no Sudeste. 


\subsubsection{Disparidades estaduais de consumo de energia comercial per capita: Comparativo entre o ano inicial e o ano final da série}

FIGURA 4- BOX-PLOT - CONSUMO DE ENERGIA COMERCIAL PER CAPITA ANO 1995 (ACIMA) E 2004 (ABAIXO)
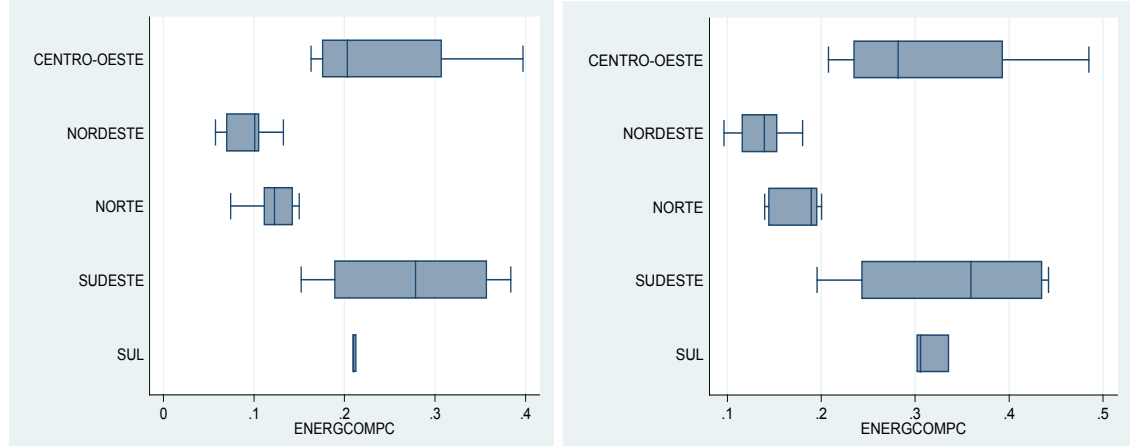

Fonte: Elaboração própria com base nos dados do IPEA, 2013.

A partir da figura 4 percebe-se que, em seu conjunto, o Sudeste apresentou nessa variável grande vantagem em relação a todas as outras macrorregiões nos anos de 1995 e 2004. Isso pode ser ilustrado pelas maiores estatísticas nos três quartis (o que inclui sua mediana, que é o segundo quartil).

Também é visível na figura 4 (em 1995 e 2004) que Sul e Centro-Oeste apresentaram medianas muito próximas entre si, com vantagem, nessa medida de centro, para o Sul. Mas, existe uma grande diferença na dispersão entre essas duas regiões: a dispersão do Sul é mínima comparada a do Centro-Oeste ou a de qualquer outra região brasileira. Observa-se, também, que todas as Unidades Federativas do Sul estão concentradas em um nível situado minimamente acima da mediana do Centro-Oeste.

\subsection{Resultados econométricos ${ }^{4}$}

Nesta seção, serão estimadas regressões baseadas nas equações 10 e 11, apresentadas na Metodologia. 
OLIVEIRA, M. T.; PAIXÃO, A. N. da. Desigualdades Regionais de Renda e as Variações nos Determinantes do....

TABELA 01 - TESTES DE COMPARAÇÃO DE MÉTODOS DE ESTIMAÇÃO

\begin{tabular}{c|c|c|c}
\hline \multicolumn{2}{c|}{ Teste LM de Breusch-Pagan ${ }^{*}$} & \multicolumn{2}{|c}{ Teste de Hausman para o Painel ** } \\
\hline chibar2(o1) & $\begin{array}{c}\text { Prob > } \\
\text { chibar2(o1) }\end{array}$ & chi2(2) & Prob >chi2 \\
\hline 405,62 & 0,0000 & 50,21 & 0,0000 \\
\hline
\end{tabular}

Fonte: Elaboração própria com base nos dados do IPEA, 2013.

* Hipótese nula: a variância de $\mathbf{C}_{\mathbf{i}}$ ser nula, o que equivale à inexistência de heterogeneidade não observada no modelo estimado, $\mathrm{H}_{\mathrm{o}}: \sigma_{\mathrm{c}}^{2}=\mathrm{O}$.

${ }^{* *}$ Hipótese nula: $\mathrm{X}_{\mathrm{it}}$ e $\mathrm{C}_{\mathrm{i}}$ não são correlacionados, $\mathrm{H}_{\mathrm{o}}: \mathrm{E}\left[\mathrm{c}_{\mathrm{i}} \mid \mathrm{x}_{\mathrm{it}}\right]=0$.

Com base no Teste LM de Breusch e Pagan, pode-se afirmar que a variância de $\mathrm{c}_{\mathrm{i}}$ não é nula, o que equivale à existência de heterogeneidade não observada no modelo estimado.

Assim, os resultados do teste de LM de Breusch-Pagan (tabela 01) favorecem a suposição de que um MQO na regressão estimada não é mais adequado do que os métodos FE e RE, que consideram a existência de heterogeneidade não observada na regressão estimada.

O teste de Hausman (1978) (tabela 01) testa a hipótese de inexistir correlação entre o efeito não-observado $c_{i}$ e as variáveis explicativas $x_{i}$ (vetor) nas regressões estimadas. Pelo resultado, pode-se rejeitar a referida hipótese, portanto, isto permite supor que existe correlação entre o efeito não-observado $c_{i}$ e as variáveis explicativas $x_{i}$ (vetor). Por consequência, o uso de FE é mais adequado, também, que o uso de RE.

O teste de multicolinearidade utilizado foi o Fator Inflação de VariânciaFIV (tabela 02). Com base nos resultados desse teste, é possível inferir que nenhuma das variáveis explicativas utilizadas neste estudo apresenta multicolinearidade entre si.

TABELA 02 - TESTE DE MULTICOLINEARIDADE NO PAINEL DE DADOS

\begin{tabular}{lc}
\hline Variáveis & FIV $^{*}$ \\
\hline $\ln$ (ENERGCOMPC) & 4,36 \\
$\ln$ ESC & 4,19 \\
$\ln$ (ENERGINDPC) & 1,28 \\
$\ln$ (crescimento pop.) & 1,14 \\
Média FIV & 2,74 \\
\hline
\end{tabular}

Fonte: Elaboração própria com base nos dados do IPEA, 2013.

*Como regra prática, se o valor do FIV for superior a dez, há sérios indícios de Multicolinearidade. 
O teste de heterocedastidade utilizado foi o Teste de Wald (tabela 03). Com base na análise dos resultados deste teste, é plausível rejeitar a hipótese nula de ausência de heterocedasticidade.

TABELA O3- TESTES DE HETEROCEDASTICIDADE E AUTOCORRELAÇÃO NO PAINEL DE DADOS

\begin{tabular}{l|c|c|c|c}
\hline \multirow{2}{*}{} & \multicolumn{2}{|c|}{$\begin{array}{c}\text { Heterocedasticidade } \\
\text { (Teste de Wald) }\end{array}$} & \multicolumn{2}{c}{$\begin{array}{c}\text { Autocorrelação } \\
\text { (teste de Wooldridge) }\end{array}$} \\
\cline { 2 - 5 } & $\chi^{2}(27)$ & Prob $>\chi^{2}$ & $\mathrm{~F}(1,26)$ & Prob $>\mathrm{F}$ \\
\hline $\begin{array}{l}\text { Conjunto de } \\
\text { variáveis A }\end{array}$ & 4622,37 & 0,0000 & 36,083 & 0,0000 \\
\hline $\begin{array}{l}\text { Conjunto de } \\
\text { variáveis B }\end{array}$ & 3879,91 & 0,0000 & 46,367 & 0,0000 \\
\hline
\end{tabular}

Fonte: Elaboração própria com base nos dados do IPEA, 2013.

* Hipótese nula: os distúrbios são homocedásticos.

** Hipótese nula: Inexiste autocorrelação de primeira ordem.

Além disso, com base no Teste de Wooldridge, aplicado na tabela 03, chega-se a conclusão de que se deve rejeitar a hipótese nula de ausência de autocorrelação. Portanto, é necessário um estimador que considere autocorrelação e heterocedastidade. O que invalida novamente possíveis análises direcionadas aos coeficientes obtidos por MQO, FE e RE, apresentados na tabela 04 .

Sendo assim, foi necessário utilizar a regressão de Prais-Winsten apresentada na tabela 04 para considerar a autocorrelação e a heterocedastidade.

Desse modo, com este último método é possível concentrar as análises sobre os coeficientes de cada determinante teórico, representados por suas respectivas variáveis proxies apresentadas anteriormente.

Dessa maneira, a regressão estimada utilizando o método de Prais-Winsten foi apresentada na tabela 04, sob a denominação PCSE(A). Destarte, com base no teste $\chi^{2}$, pode-se rejeitar a hipótese que todos os coeficientes estimados são simultaneamente iguais à zero, sob um nível de significância de $1 \%$.

Além disso, a partir da apuração dos resultados do teste $\mathrm{z}$, na regressão PCSE(A), constata-se que apenas o coeficiente associado a variável "taxa de crescimento populacional" não é significativo, recorda-se que a referida variável é uma proxy para taxa de depreciação efetiva do capital. Ressalta-se que os demais coeficientes são todos significativos sob um nível de 1\%, segundo o teste $\mathrm{z}$. 
OLIVEIRA, M. T.; PAIXÃO, A. N. da. Desigualdades Regionais de Renda e as Variações nos Determinantes do....

TABELA 04 - RESULTADOS DOS MODELOS DE REGRESSÃO (EQUAÇÕES 10 E 11)

\begin{tabular}{|c|c|c|c|c|c|}
\hline \multirow{2}{*}{ Variáveis } & \multicolumn{5}{|c|}{ Variável dependente: ln do PIB per capita } \\
\hline & POLS & $\mathrm{FE}$ & $\mathrm{RE}$ & PCSE(A) & $\operatorname{PCSE}(\mathrm{B})$ \\
\hline \multirow[t]{2}{*}{$\operatorname{lnESC}$} & $2,11983^{* * *}$ & $0,80432^{* * *}$ & $1,12728 * * *$ & $1,750549^{* * *}$ & $0,5556188 * * *$ \\
\hline & $(0,07747)$ & $(0,09991)$ & $(0,09658)$ & $(0,0958441)$ & $(0,1290226)$ \\
\hline \multirow[t]{2}{*}{ lnENERGINDP } & $0,13319^{* * *}$ & $0,10446^{* * *}$ & $0,11777^{* * *}$ & $0,1301186^{* * *}$ & \\
\hline & $(0,01412)$ & $(0,03064)$ & $(0,02438)$ & $(0,0208404)$ & \\
\hline \multirow[t]{2}{*}{ lnENERGCOMP } & & & & & $0,7969761^{* * *}$ \\
\hline & & & & & $(0,055003)$ \\
\hline \multirow[t]{2}{*}{ Ln cresc. Pop } & $-1,39637^{*}$ & 0,47283 & 0,44682 & $-0,3355406$ & $-0,0997696$ \\
\hline & $(0,80927)$ & $(0,42771)$ & $(0,46600)$ & $(0,5844218)$ & $(0,3674773)$ \\
\hline \multirow[t]{2}{*}{ Constante } & $-1,87877^{* * *}$ & 0,25873 & $-0,26748$ & $-1,268921^{* * *}$ & $1,961045^{* * *}$ \\
\hline & $(0,13047)$ & $(0,17862)$ & $(0,17301)$ & $(0,174704)$ & $(0,2945426)$ \\
\hline $\mathrm{N}$ & 270 & 270 & 270 & 270 & 270 \\
\hline $\mathrm{R}^{2}$ & 0,77311 & 0,27544 & & 0,9041 & o,8912 \\
\hline $\mathrm{R}^{2}$ (overall) & & 0,72057 & 0,74901 & & \\
\hline $\mathrm{R}^{2}$ (between) & & 0,78010 & 0,81579 & & \\
\hline R²(within) & & 0,27544 & 0,27387 & & \\
\hline $\mathrm{F}$ & $3,0 \mathrm{e}+02$ & 30,41256 & & & \\
\hline chi2 & & & $1,8 \mathrm{e}+02$ & 602,65 & 917,15 \\
\hline Prob > F & 0,0000 & 0,0000 & & & \\
\hline Prob > chi2 & & & 0,0000 & 0,0000 & 0,0000 \\
\hline Chow $F(26,240)$ & & 35,38 & & & \\
\hline Chow Prob $>F$ & & 0,0000 & & & \\
\hline
\end{tabular}

Fonte: Elaboração própria com base nos dados do IPEA, 2013.

Erros-padrão em parêntesis. ${ }^{* *}$ Significante a $1 \%$; ${ }^{* *}$ Significante a $5 \%$; ${ }^{*}$ Significante a 10\%, com base no teste t.

Estimadores:

POLS: POLS, Mínimos Quadrados Ordinários (MQO);

FE: Efeitos Fixos;

RE: Efeitos Aleatórios;

PCSE(A): Regressão de Prais-Winsten (conjunto de variáveis A)

PCSE(B): Regressão de Prais-Winsten (conjunto de variáveis B)

Variável dependente: Logaritmo natural do PIB per capita. Variáveis explicativas: Logaritmo natural da média dos anos de estudo (lnESC); Logaritmo natural do consumo de energia industrial per capita (lnENERGINDPC); Logaritmo natural do consumo de energia comercial per capita (lnENERGCOMPC);Logaritmo natural da taxa de crescimento populacional (Ln cresc. Pop).

Ademais, é relevante observar que o $\mathrm{R}^{2}$ da regressão PCSE(A) indica que aproximadamente 90,41\% das variações na variável dependente são explicadas pelo modelo estimado a partir das variáveis escolhidas. Como estas representam os determinantes do produto per capita de longo prazo sugeridos pelo referencial 
teórico utilizado neste trabalho, o valor desse indicador é mais um indício que confirma que as diferenças nestes determinantes possuem sérias implicações no cenário das desigualdades regionais de renda existente no Brasil.

É importante pontuar que foi testada outra proxy para capital físico no lugar da proxy utilizada na regressão PCSE(A). Para tanto, foi substituído o "consumo de energia elétrica no setor industrial" pelo "consumo de energia elétrica no setor comercial”, esta especificação alternativa está identificada na tabela 04 como regressão PCSE(B).

Ainda na tabela 04, repara-se que os resultados dos testes $\chi^{2}$ e z da regressão PCSE(B) permitiram as mesmas conclusões que foram obtidas anteriormente, no entanto, o resultado do $\mathrm{R}^{2}$ dessa nova regressão foi reduzido para $89,12 \%$. Embora este resultado ainda seja muito satisfatório, este significa que a regressão PCSE(B) possui um poder de explicação menor do que a regressão PCSE(A).

Por conseguinte, a proxy "consumo de energia elétrica no setor industrial" foi utilizada na regressão escolhida (PCSE(A)) para as analises dos coeficientes estimados (elasticidades), considerando uma amostra com as 27 unidades federativas brasileiras.

Observa-se, ainda, que todos os coeficientes estimados para as variáveis explicativas apresentaram os sinais esperados de acordo com a teoria econômica.

Em termos de elasticidades estimadas destaca-se que, a partir da tabela 04, para cada $1 \%$ de aumento na variável escolaridade (média de anos de estudo) tem-se uma variação positiva de $1,75 \%$ no PIB per capita das unidades federativas brasileiras, enquanto que um acréscimo de $1 \%$ na variável proxy de capital físico impacta em uma variação positiva de $0,13 \%$ no PIB per capita das unidades federativas brasileiras.

Estas elasticidades confirmam a grande importância de aumentos nos níveis de escolaridade para o crescimento econômico de uma região, sendo que estes apresentam um impacto maior na renda das regiões do que o investimento em capital físico, que também possui impacto positivo na determinação do produto per capita dos estados brasileiros.

Consequentemente, estas evidências favoráveis à importância do capital humano e do capital físico na determinação do produto per capita das regiões brasileiras, aliadas à descrição estatística realizada no presente estudo, possibilitam concluir que as desigualdades regionais de renda no Brasil são, em grande medida, reflexos das desigualdades regionais nos determinantes explorados no referencial teórico deste trabalho, confirmando o que se tornava aparente, porém, menos conclusivo do que agora, na seção de estatística descritiva.

Pois, como demonstrado naquela seção, existiam pronunciadas desigualdades 
regionais nos determinantes, favoráveis às regiões mais ricas do país. Deste modo, uma contribuição da análise econométrica, apresentada na presente seção, é confirmar a relação de causa e efeito existente entre essas desigualdades nos determinantes e as disparidades regionais de renda brasileiras.

No entanto, os resultados dos testes F de Chow (tabela 04), LM de Breusch-Pagan (tabela 01) e Hausman (1978) (tabela 01), ainda constituem indícios de que existe um efeito não-observado influenciando a variável dependente, e de que este é correlacionado com as variáveis explicativas da regressão, porém, os problemas de autocorrelação e a heterocedastidade impediram a utilização de FE, e foi necessário utilizar os estimadores do método de Prais-Winsten.

TABELA O5 - RESULTADOS DOS MODELOS DE REGRESSÃO, PARA GRUPOS REGIONAIS (EQUAÇÕES 10 E 11)

\begin{tabular}{l|c|c|c|c}
\hline \multirow{2}{*}{ Variáveis } & \multicolumn{4}{|c}{ Variável dependente: ln do PIB per capita } \\
\cline { 2 - 5 } & PCSE(A) NNO & $\begin{array}{c}\text { PCSE(B) } \\
\text { NNO }\end{array}$ & $\begin{array}{c}\text { PCSE(A) } \\
\text { CSUL }\end{array}$ & $\begin{array}{c}\text { PCSE(B) } \\
\text { CSUL }\end{array}$ \\
\hline lnESC & $\begin{array}{c}1,11889^{* * *} \\
(0,1084163)\end{array}$ & $0,5326174^{* * * *}$ & $2,108736^{* * *}$ & $1,626224^{* * *}$ \\
lnENERGINDPC & $0,0362716^{* *}$ & $0,1312772)$ & $(0,1708251)$ & $(0,2489007)$ \\
& $(0,0169294)$ & 0,0420857 & \\
lnENERGCOMPC & & $0,4942137^{* * *}$ & $(0,0317238)$ & \\
Ln cresc. pop & $-0,098904$ & $-0,1652125$ & $-1,324024^{* *}$ & $-1,081551^{*}$ \\
Constante & $(0,5429576)$ & $(0,4109318)$ & $(0,6491009)$ & $(0,6276189)$ \\
& $-0,5572706^{* * *}$ & $1,326012^{* * *}$ & $-1,750457^{* * *}$ & $-0,5887871$ \\
N & $(0,1615841)$ & $(0,3312869)$ & $(0,3117554)$ & $(0,5531228)$ \\
R2 & 160 & 160 & 110 & 110 \\
chi2 & 0,8991 & 0,8057 & 0,9489 & 0,9473 \\
Prob > chi2 & 114,49 & 146,80 & 204,89 & 134,58 \\
\hline
\end{tabular}

Fonte: Elaboraçao propria com base nos dados do IPEA, 2013.

Erros-padrão em parêntesis. ${ }^{* * *}$ Significante a $1 \%$; ** Significante a $5 \%$; * Significante a $10 \%$, com base no teste z.

Estimadores:

PCSE(A): Regressão de Prais-Winsten (conjunto de variáveis A);

PCSE(B): Regressão de Prais-Winsten (conjunto de variáveis B);

NNO: Grupo NNO (grupo 1) compreende os estados do Norte e do Nordeste;

CSUL: Grupo CSUL (grupo 2) compreende os estados do Centro-Oeste, Sudeste e Sul;

Variável dependente: Logaritmo natural do PIB per capita. Variáveis explicativas: Logaritmo natural da média dos anos de estudo (lnESC); Logaritmo natural do consumo de energia industrial per capita (lnENERGINDPC); Logaritmo natural do consumo de energia comercial per capita (lnENERGCOMPC); Logaritmo natural da taxa de crescimento populacional (Ln cresc. Pop). 
Não obstante, para os propósitos deste trabalho uma alternativa se mostra viável para reduzir a heterogeneidade regional: estimar regressões separadas para diferentes regiões, considerando os níveis das variáveis explicativas e da variável dependente. Desta maneira, as cinco macrorregiões do país foram agrupadas em duas, de acordo com as suas realidades econômicas.

Com base nisto, foram definidos os seguintes grupos regionais: o grupo NNO (grupo 1) que compreende os estados do Norte e do Nordeste; e o grupo CSUL (grupo 2) que compreende os estados do Centro-Oeste, Sudeste e Sul.

Assim, será possível verificar possíveis variações nos parâmetros e nos ajustamentos das regressões, com intuito de captar diferenças econômicas, entre as regiões, referentes aos impactos que cada determinante possui sobre o PIB per capita estadual.

Primeiro, foi estimada uma regressão para o grupo NNO (tabela 05) utilizando as mesmas variáveis explicativas usadas na regressão PCSE(A). Desta forma, com base no teste $\chi^{2}$ (tabela 05), é possível rejeitar a hipótese que todos os coeficientes estimados são simultaneamente iguais à zero, sob um nível de significância de $1 \%$.

Além de que, foi verificado, com base no teste z, que apenas o coeficiente da variável "taxa de crescimento populacional" não foi significativo, mas todos os demais coeficientes estimados são estatisticamente diferentes de zero, sendo que este resultado para o coeficiente de escolaridade, e para a constante, está sob um nível de significância de $1 \%$, enquanto o resultado para o coeficiente de capital físico está sob um nível de significância de $5 \%$.

É importante sublinhar que o $\mathrm{R}^{2}$ da regressão "PCSE(A) NNO” indica que aproximadamente $89,91 \%$ das variações na variável dependente são explicadas pelo modelo estimado, com base no conjunto de variáveis escolhidas. Como estas representam os determinantes do produto per capita de longo prazo sugeridos pelo referencial teórico utilizado neste trabalho, o valor desse indicador é mais um indício que confirma que as diferenças nestes determinantes também possuem sérias implicações no cenário das desigualdades regionais de renda que existem entre os estados das regiões Norte e Nordeste.

Posteriormente, também para o grupo NNO, foi testado outra proxy para capital físico no lugar da proxy utilizada na regressão "PCSE(A) NNO". Desta maneira, foi substituído o "consumo de energia elétrica no setor industrial" pelo "consumo de energia elétrica no setor comercial", ressalta-se que esta especificação alternativa está identificada na tabela 05 como regressão "PCSE(B) NNO".

Deste modo, avista-se na tabela 05 que os resultados dos testes $\chi^{2}$ e z da regressão "PCSE(B) NNO" permitiram as mesmas conclusões que foram obtidas anteriormente, no entanto, o resultado do $\mathrm{R}^{2}$ dessa nova regressão foi reduzido para $80,57 \%$. Mesmo que este resultado ainda seja muito satisfatório, 
este significa que esta nova especificação "PCSE(B) NNO" possui um poder de explicação menor do que a anterior "PCSE(A) NNO", da mesma forma que ocorreu para a amostra que considerava todas as unidades federativas do país.

Portanto, a proxy "consumo de energia elétrica no setor industrial" foi utilizada na regressão que foi escolhida (PCSE(A) NNO) para as análises dos coeficientes estimados (elasticidades), considerando uma amostra com os dezesseis estados das regiões Norte e Nordeste.

Observa-se, ainda, que todos os coeficientes estimados na regressão "PCSE(A) NNO” para as variáveis explicativas apresentaram os sinais esperados de acordo com a teoria econômica.

Referente às elasticidades estimadas aponta-se que, a partir da tabela 05 , para cada $1 \%$ de aumento na variável escolaridade (média de anos de estudo) tem-se uma variação positiva de $1,12 \%$ no PIB per capita das unidades federativas consideradas, enquanto que um acréscimo de $1 \%$ na variável proxy de capital físico impacta em uma variação positiva de 0,04\% no PIB per capita das unidades federativas do grupo NNO.

Tais elasticidades confirmam, mais uma vez, a grande importância de aumentos nos níveis de escolaridade para o crescimento econômico de uma região, pois estes possuem um impacto no PIB per capita estadual maior do que o impacto do estoque de capital físico, que também possui impacto positivo na determinação do produto per capita nos estados do Norte e do Nordeste.

O passo seguinte foi estimar uma regressão para o grupo CSUL (tabela 05; coluna "PCSE(A) CSUL") utilizando as mesmas variáveis explicativas usadas na regressão PCSE(A). Desta forma, com base no teste $\chi^{2}$ (tabela 05), é possível rejeitar a hipótese que todos os coeficientes estimados são simultaneamente iguais à zero, sob um nível de significância de $1 \%$.

Entretanto, a partir do teste z, verificaram-se duas importantes diferenças deste grupo regional (CSUL) frente ao grupo NNO, e também em relação à regressão que usou a amostra nacional. Uma dessas diferenças refere-se ao fato de que a proxy "consumo de energia elétrica do setor industrial" não apresentou valor significante para o seu coeficiente nem a 10\% de significância, na regressão "PCSE(A) CSUL".

Isso sugere que ao retirar da amostra os estados das macrorregiões mais pobres e com menor capital físico industrial, tal variável deixar de explicar a variação do PIB per capita que ocorre entre as UFs do grupo CSUL e ao longo do tempo para essas mesmas unidades. Portanto, tal fato pode estar ligado aos rendimentos marginais decrescentes do capital físico industrial, visto que os estados dessas macrorregiões possuem maior estoque dessa variável.

A outra particularidade do grupo CSUL é que, neste caso, o coeficiente associado à variável "taxa de crescimento populacional", representando a taxa 
de depreciação efetiva do capital físico, foi significante sob um nível de $5 \%$. Dado que o impacto sobre o PIB per capita verificado para essa variável foi negativo, percebe-se que somente nesse grupo regional a previsão do modelo de Solow de que um maior crescimento populacional está associado a uma renda per capita menor é confirmada.

Ainda pelo teste z, relata-se que o coeficiente da variável escolaridade manteve seu valor significante até a 1\% de significância, assim como nas regressões anteriores.

Apesar da proxy de capital físico não ter sido considerada significante, acentua-se que o $\mathrm{R}^{2}$ da regressão "PCSE CSUL(A)" indica que aproximadamente 94,89\% das variações na variável dependente são explicadas pelo modelo estimado.

Seguidamente, também para o grupo CSUL, foi testado outra proxy para capital físico no lugar da proxy estimada pela regressão "PCSE(A) CSUL". Destarte, foi substituído o "consumo de energia elétrica no setor industrial" pelo "consumo de energia elétrica no setor comercial", obtendo-se uma especificação identificada na tabela 05 por "PCSE(B) CSUL".

Repetidamente, o resultado do teste $\chi^{2}$ da regressão "PCSE(B) CSUL" permitiu a mesma conclusão que foi obtida anteriormente, no entanto, o resultado do $\mathrm{R}^{2}$ dessa nova regressão sofreu uma pequena redução para 94,73\%. Portanto, este resultado significa que esta nova especificação possui um poder de explicação menor do que a anterior.

Isto posto, a proxy "consumo de energia elétrica no setor industrial" foi utilizada na regressão escolhida (PCSE(A) CSUL) para as análises dos coeficientes estimados (elasticidades), considerando uma amostra com as onze unidades federativas das regiões Centro-Oeste, Sudeste e Sul.

Na sequência, atenta-se que todos os coeficientes estimados para as variáveis explicativas apresentaram os sinais esperados de acordo com a teoria econômica.

Com respeito às elasticidades estimadas frisa-se, a partir da regressão "PCSE(A) CSUL" na tabela 05, que para cada $1 \%$ de aumento na variável escolaridade (média de anos de estudo) tem-se uma variação positiva de 2,11\% no PIB per capita das unidades federativas consideradas, enquanto que um acréscimo de $1 \%$ na variável "taxa de crescimento populacional", impacta em uma variação negativa de 1,32\% no PIB per capita estadual do grupo CSUL.

Estas elasticidades confirmam a grande importância de aumentos nos níveis de escolaridade para o crescimento econômico da região considerada. Por outro lado, recorda-se que, na regressão "PCSE(A) CSUL", não foi significativo o coeficiente estimado para o capital físico, representado pela proxy "consumo de energia elétrica no setor industrial”. 
Diante dos resultados econométricos apresentados (tabelas 04 e 05), pretende-se, a partir daqui, retomar algumas dessas informações de modo a realizar novas comparações entre os ajustamentos obtidos para cada grupo regional considerado, e também, entre as magnitudes das elasticidades estimadas para diferentes regiões do país.

À vista disso, recapitula-se que as variáveis consideradas significativas, a partir do teste z, na regressão "PCSE(A) NNO" foram as seguintes: "média de anos de estudo", "consumo de energia industrial". Enquanto que a variável "taxa de crescimento populacional" não foi considerada significativa na referida especificação.

Concernente à regressão "PCSE(A) CSUL", as variáveis consideradas significativas, a partir do teste $\mathrm{z}$, foram as seguintes: "média de anos de estudo", "taxa de crescimento populacional". Por outro lado, a variável "consumo de energia industrial” não foi considerada significativa para tal especificação.

Em virtude dessas diferenças ligadas aos coeficientes, não será possível comparar as elasticidades relacionadas à variável capital físico, caso se considere somente as especificações com o "conjunto A" de variáveis, como foi descrito anteriormente. Então, serão confrontados os coeficientes obtidos pelas especificações que consideram o "conjunto A" e, também, pelas especificações que consideram o "conjunto B", pois desta maneira, será possível comparar os efeitos tanto de capital humano, quanto de capital físico.

Assim, a figura 05 foi feita com base na especificação A, enquanto que a figura 06 foi feita com base na especificação B.

Pela figura 05, nota-se que a elasticidade da escolaridade na regressão do CSUL é muito superior à mesma medida obtida na regressão do NNO, sendo que o valor obtido para o primeiro grupo corresponde a quase o dobro do segundo grupo. Para a amostra nacional foi encontrado um valor que fica entre os valores obtidos para os dois cortes regionais analisados. Em outras palavras o acréscimo de escolaridade contribui mais para o PIB per capita estadual no grupo CSUL que no Grupo NNO, o que se constitui em mais uma vantagem para as regiões mais ricas do país, que também possuem vantagem quanto à dotação dessa variável. Ressalta-se, com base na figura 05, que o impacto da variável "escolaridade" na renda é muito maior que o impacto da outra variável, sendo assim, diferenças relacionadas a essa variável implicam, proporcionalmente, em maior hiato regional de PIB per capita. 
FIGURA o5 - COEFICIENTES CALCULADOS NAS REGRESSÕES “PCSE(A)”, "PCSE(A) NNO”, "PCSE(A) CSUL"

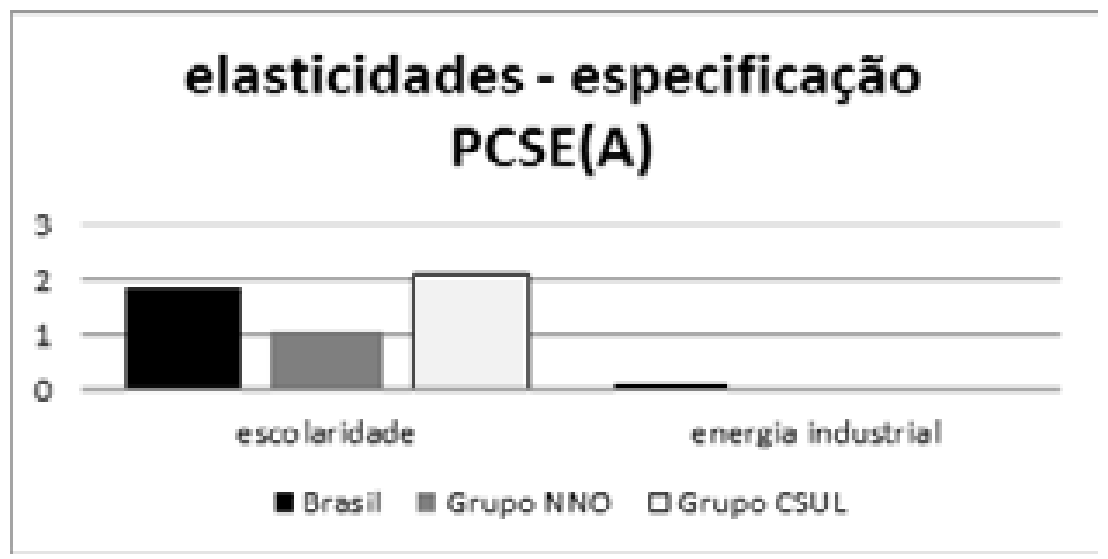

Fonte: Resultados da tabela 04 (regressão PCSE(A)) e da tabela 05 (regressão "PCSE(A) NNO" e regressão “PCSE(A) CSUL”)

Com relação à elasticidade do capital físico, observa-se pela figura 05 que o valor correspondente à regressão com a amostra nacional possui um valor muito maior ao valor obtido para o grupo NNO (mais de três vezes maior). Já o valor do grupo CSUL foi considerado igual à zero em razão do resultado do teste $\mathrm{z}$.

Isso mostra que ao retirar da amostra nacional o grupo CSUL, cai o impacto que variável capital físico possui na explicação da variação de PIB per capita que ocorre entre as UFs e ao longo do tempo nessas unidades. Dado que dentro do grupo CSUL tal variável não foi significativa e que há grandes diferenças de dotações dessa variável favoráveis ao grupo CSUL, conclui-se que a significância dessa variável na amostra nacional ocorre, em grande parte, devido ao hiato existente entre o NNO e CSUL na variável dependente (capital físico) e explicativa.

Pela figura 06, repetidamente, avista-se que a elasticidade da escolaridade na regressão do CSUL é muito superior à mesma medida obtida na regressão do $\mathrm{NNO}$, sendo que o valor obtido para o primeiro grupo corresponde a quase o triplo do segundo grupo. Para a amostra nacional foi encontrado um valor que fica entre os valores obtidos para os dois cortes regionais analisados.

Com relação à elasticidade do capital físico, capta-se pela figura 06 , que o valor correspondente à regressão do NNO possui um valor superior ao valor obtido para o grupo CSUL. Para a amostra nacional foi encontrado um valor maior que os valores correspondentes aos dois cortes regionais analisados. Tendo em vista que o grupo CSUL possui estoques mais elevados para essa variável do que o grupo NNO, além de maior média de renda per capita, sugere-se 
aqui que essa redução de impactos dessa variável seja decorrente da ação dos rendimentos marginais decrescentes do capital físico em questão.

FIGURA o6 - COEFICIENTES CALCULADOS NAS REGRESSÕES "PCSE(B)”, "PCSE(B) NNO”, "PCSE(B) CSUL"

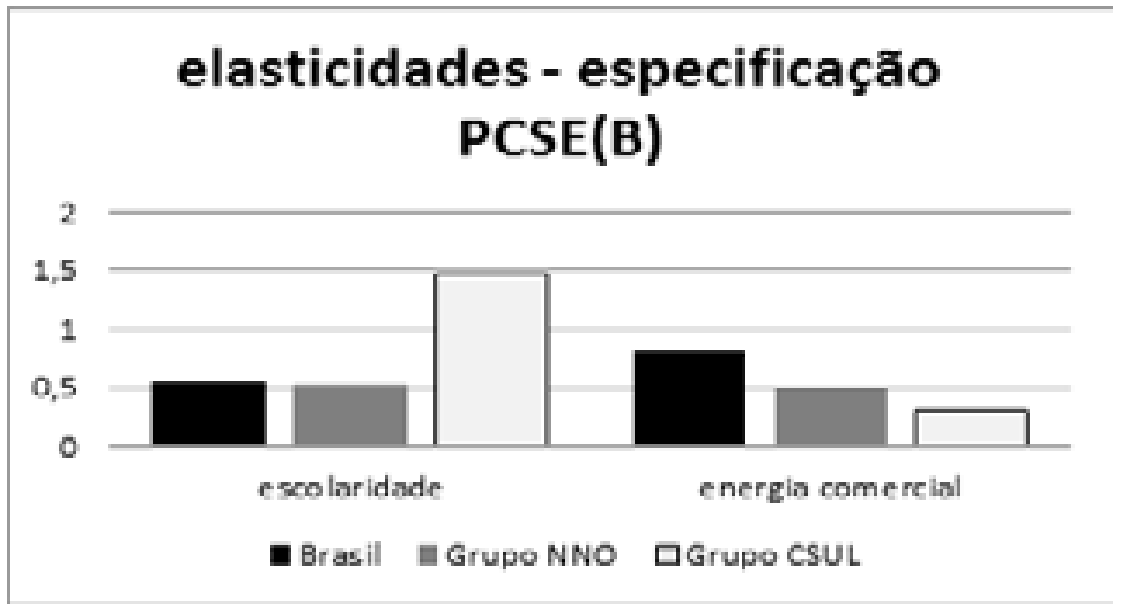

Fonte: Resultados da tabela 04 (regressão PCSE(B)) e da tabela 05 (regressão "PCSE(B) NNO" e regressão “PCSE(B) CSUL”)

\section{Conclusões}

Os resultados apresentados possibilitam concluir que as desigualdades regionais de renda no Brasil são, em grande medida, reflexos das disparidades regionais nas dotações de capital físico e capital humano, o que é coerente com a função de produção proposta por MRW (1992).

Todavia, os grandes contrastes entre as macrorregiões do país não são oriundos somente de distinções nas suas dotações de fatores de produção. Uma vez que também foram constatadas grandes diferenças nos efeitos que a variável capital humano tem sobre o PIB per capita, favoráveis às macrorregiões mais ricas, que são Sudeste, Sul e Centro-Oeste.

Por outro lado, o efeito estimado que a variável capital físico tem sobre a renda é superior nas macrorregiões Norte e Nordeste em relação ao outro agrupamento regional. No entanto, esse diferencial não reverte à desvantagem total dessas macrorregiões por que o mesmo foi bem menor que a diferença vista na outra variável. Além de que, os impactos estimados sobre a renda decorrentes de acréscimos na variável capital físico foram inferiores aos calculados 
para a outra variável, em todas as especificidades e cortes regionais testados.

Destarte, existem no Brasil consideráveis variações regionais tanto nos efeitos quanto nas dotações das variáveis consideradas determinantes da renda per capita de longo prazo. Esses dois tipos de variações reforçam, de forma conjunta, as desvantagens das regiões mais pobres (Norte e Nordeste), o que leva a concluir que existem grandes diferenças nas condições estruturais das economias dos estados e macrorregiões do país.

Dado que tais condições necessitam de longos prazos para se transformarem, não se vislumbra, pelos dados analisados, redução expressiva das mencionadas desigualdades de renda dentro de curto ou médio prazo. Isto é, pelas tendências expostas neste estudo não foram constatados aspectos de cunho transitório no cenário econômico regional brasileiro.

Diante do exposto, é imprescindível o debate acerca de quais políticas públicas são mais eficazes no sentido de possibilitar e incentivar mais educação e investimentos em cada uma das vinte e sete unidades federativas brasileiras. Visto que, tais políticas se constituem caminhos de desenvolvimento econômico viáveis para todo o país, em especial para as regiões mais pobres, que possuem mais carências em relação às essas variáveis.

Em vista disso, tornam-se valiosos novos estudos que busquem entender as trajetórias das regiões mais desenvolvidas do país, inclusive com estudos comparados, com a finalidade de verificar quais lições que as demais regiões podem tirar dessas trajetórias.

\section{Referências}

Azzoni, C. R., Menezes-Filho, N., Menezes, T., \& Silveira-Neto, R. (2000). Geography and income convergence among Brazilian states. Research Network Working Paper R-395, Inter-American Development Bank, Washington DC.

Azzoni, C. R; Menezes Filho, N.; Menezes, T. A.; Silveira Neto, R. M. (2004). Geography and income growth accross Brazilian states: evidence from cohort data. Journal of Regional Science.

Chaves, M. C. Examinando as desigualdades regionais: Um teste de convergência para a renda per capita familiar brasileira, 1970-1991. (2003). Curitiba: Dissertação (Mestrado em Desenvolvimento Econômico), Universidade Federal do Paraná.

Chagas, André Luís Squarize; TONETO Jr, Rudinei. (2003). Fatores determinantes do crescimento local - evidências a partir de dados dos municípios brasileiros para o período 1980-1991. Pesquisa e Planejamento Econômico - PPE. Vol. $33-n .^{\circ} 2$.

Ferreira, A. A distribuição interestadual da renda no Brasil, 1950-85. (1996) Revista Brasileira de Economia, Brasil, v. 50.

Ferreira, A. H. B. Convergence in Brazil: Recent trends and long-run prospects. 
OLIVEIRA, M. T.; PAIXÃO, A. N. da. Desigualdades Regionais de Renda e as Variações nos Determinantes do....

(2000). Applied Economics, v. 32, p. 479-489.

Greene, W.h. Econometric analysis. (2001). 4th ed. New Jersey: Prentice-Hall, $1075 p$.

Hausman, J.a. (1978) Specification tests in econometrics. Econometrica, v. 46, $n$. 6, p. 1251-1271.

IPEA - Instituto de Pesquisa Econômica Aplicada. Disponível em: <http://www. ipeadata.gov.br>. Acesso em: 30 setembro de 2013.

Jones, Charles I. (2000). Introdução à teoria do crescimento econômico; tradução de Maria José Cyhlar Monteiro. - Rio de Janeiro: Elsevier, $19^{a}$ reimpressão.

Mankiw, N. G.; Romer, D.; Weil, D. (1992). A contribution to the empirics of economic growth. The Quarterly Journal of Economics, v. 107, n. 2. p. 407- 437.

Ribeiro, E. C. B. A.; Goncalves, E.; Freguglia, R. S. (2013). Transbordamentos de Tecnologia e Capacidade de Absorção: uma Análise para os Estados Brasileiros. Revista ANPEC.

Resende, G. M. \& Figueiredo, L. (2005). Testes de robustez: Uma aplicação para os determinantes das taxas de crescimento do produto interno bruto per capita dos estados brasileiros. Texto para Discussão 1124. Brasília: IPEA.

Silveira-Neto, R. \& Azzoni, C. R. (2005). Decomposing regional growth: Labor force participation rates, structural changes, and sectoral factor reallocation. The Annals of Regional Science, 39(2), p. 221-239.

Solow, Robert M. (1956). A contribution to the theory of economic growth. The Quarterly Journal of Economics, v. 70, n. 1, p. 65-94.

Wooldridge, Jeffrey M. (2002). Econometric Analysis of Cross Section and Panel Data. The MIT Press, Cambridge, MA. 